\title{
CÁNCER DE MAMA EN CHILE. MORTALIDADES COMPARADAS: NIVELES NACIONAL, REGIONAL Y COMUNAL, 1990-2011. ANÁLISIS PROGRAMÁTICO*
}

\author{
Dr. Iván Serra C. ${ }^{1}$, Daysi Maya G. ${ }^{2}$, Dra. Claudia Aguayo S. ${ }^{3}$ \\ 1 Cirujano y epidemiólogo, Servicios de Cirugía y Urgencia, Hospital Sótero del Río, Santiago. \\ 2 Matrona, Hospital Sótero del Río, Santiago. \\ 3 Magíster en Salud Pública, epidemióloga, Hospital Regional, Concepción. \\ Chile.
}

\section{Abstract \\ National and regional mortality rates due to breast cancer in Chile from 1990 to 2011}

Background: Population or geographical differences in prevalence are observed for many diseases. These variations may give clues about the causes of the diseases. Aim: To study the geographical distribution of mortality for breast cancer in Chile. Material and Methods: Information was obtained from databases available at the Chilean Ministry of Health and the National Institute of Statistics. Crude and adjusted mortality rates during the period 1990-2011 were calculated according to quinquennial age groups, for two municipalities of Metropolitan Santiago. Results: In the study period, the crude mortality rates increased by $20 \%$, from 12-13/100,000 women in 1990 to $15.7 / 100,000$ women in 2009. Mortality is decreasing in women aged 30 to 49 years. This is not the case for women aged 25 to 29 years. Mortality is higher in La Florida Municipality. In South-Oriental Santiago, the frequency of advanced stages of the disease (III or IV), which initially were reduced to $20 \%$, have steadily increased to $28.3 \%$ in 2009 , a figure similar to the national frequency of 29 to $30 \%$. This higher frequency of advanced stages coincides with the rise in mortality. Conclusions: The increasing mortality due to breast cancer would be secondary to the high frequency of advanced stages and population aging. However, older women are not considered in the national breast cancer program. Their inclusion should be considered.

Key words: Breast cancer, mortality, regional differences.

\section{Resumen}

Introducción: Son habituales las variaciones de frecuencia geográfico-poblacionales en muchas enfermedades y países o regiones, diferencias que pueden orientar sobre sus causas. En Chile faltan estudios recientes sobre cáncer de mama con esta perspectiva. Material y Método: Estudio descriptivo-analítico de

*Recibido el 24 de julio de 2013 y aceptado para publicación el 12 de agosto de 2013.

Los autores no refieren conflictos de interés.

Correspondencia: Dr. Iván Serra C.

Barros Errázuriz 1992. Depto. 41. Providencia, Santiago, Chile.

ivan.serra.canales@gmail.com 
mortalidad para niveles nacional: 2011 (8.711.546 mujeres), regional o Servicio de Salud Sur-Oriente (SSMSO) (800.341) y dos comunas, Puente Alto y La Florida (374.886 y 199.243). Se analizaron tasas crudas, ajustadas y específicas por grupos quinquenales de edad, últimos 22 años (1990-2011). Resultados: Tasa cruda de mortalidad nacional sube en forma constante e importante (20\%), de 12-13 por 100.000 hasta 15,7 por 100.000 mujeres (2009). Tasas crudas del SSMSO son más bajas. Mortalidad por cáncer mamario está disminuyendo en las mujeres jóvenes (30-34; 35-39; 40-44; 45-49), excepto las muy jóvenes (25-29) en ambos niveles: nacional y regional. Mortalidad comunal es más alta en comuna de La Florida. Estadíos avanzados III y IV que habían descendido en el SSMSO hasta bajo $20 \%$ del total de pacientes han ido subiendo hasta $28,3 \%$ $(22,4+5,9)$ el 2009, cercano al nivel nacional estable del 29-30\%. Esto coincide con alza de su mortalidad, además de hacerse próxima a la del país, comprobando hipótesis. Conclusiones: La creciente mortalidad por cáncer de mama en Chile derivaría de alta frecuencia mantenida de estadíos avanzados y del envejecimiento poblacional. Sin embargo, las mujeres senescentes no están consideradas en el programa nacional. Debería ampliarse pesquisa de este cáncer elevando edad a través de las enfermeras en la atención primaria y estudiar causas del aumento de estadíos avanzados en el SSMSO y sus diferencias comunales.

Palabras clave: Cáncer de mama, epidemiología, variaciones geográficas, programas de salud, Chile.

\section{Introducción}

El cáncer de mama presenta grandes variaciones geográficas tanto entre continentes, como países y también regiones al interior de ellos ${ }^{1}$. Este cáncer presenta baja incidencia y mortalidad en Asia, mientras es muy alta en Europa Occidental, Estados Unidos, Canadá, Australia y Nueva Zelanda, en general en poblaciones con elevado desarrollo social y económico ${ }^{2}$. Sin embargo, existen otros factores: por ejemplo en este último país, la incidencia y especialmente la mortalidad por esta causa es bastante más alta en la población maorí que en la no maorí, ambas con niveles de vida relativamente similares ${ }^{3}$. Un caso contrario es Estados Unidos donde es máxima en las mujeres blancas con mejor nivel socio-económico, menor con origen africano, aún más baja en latinas o hispánicas (menos de la mitad comparado con las definidas como blancas) y finalmente amerindias ${ }^{4}$. La variación en el tiempo de sus tasas ajustadas de mortalidad en estos dos países es a la franca disminución en sus grupos con mayor mortalidad: maoríes en Nueva Zelanda y blancas en Estados Unidos ${ }^{2,5}$. Las causas de las diferencias entre países pueden ser tanto genéticas (raciales) como ambientales incluyendo el sistema de salud y su accesibilidad, o inherentes a las personas como culturales y otras aunque más probablemente responde a una interacción entre ellas ${ }^{6,7}$. Es conocido de antiguo que mujeres japonesas, con muy baja frecuencia de cáncer de mama en su país, al emigrar a Estados Unidos tienen hijas con mayor incidencia y nietas con frecuencia aun mayor ${ }^{8}$.

En Chile, es difícil trabajar con incidencia por carecer de registros confiables ${ }^{9-11}$. Los datos de publicaciones internacionales como Globocan sobre cáncer de mama en nuestro país son estimaciones y restringidas al sistema público de salud ${ }^{12}$. En cambio, la mortalidad es real y creciente, mostrando importantes variaciones geográficas, mantenidas en el tiempo ${ }^{13-15}$. Los datos de mortalidad incluyen el sistema de salud privado y además son confiables $^{16-18}$.

En nuestro país se han observado siempre diferencias regionales en la mortalidad por este cáncer. Un estudio de 1981, mostró la mortalidad más alta en las regiones más pobladas: Metropolitana y Valparaíso $^{13}$. Otro más reciente encontró la más elevada en el Servicio Central y la más baja en Llanquihue ${ }^{14}$. Otro estudio analizó la mortalidad por este cáncer en la región del Bío-Bío, tratando de relacionarla con factores socio-demográficos, geopolíticos y geográficos sin encontrar asociaciones positivas o negativas ${ }^{15}$. Un estudio muy reciente sobre la región metropolitana confirmó su mortalidad más elevada que lo nacional pero mostrando que eso ocurre sólo en cinco de sus seis servicios de salud ${ }^{10}$. Estadísticas actuales señalan una alta mortalidad en los tres Servicios de Salud de la región de Valparaíso, junto con Santiago ${ }^{19}$. La mortalidad por cáncer de mama es baja en Atacama y otras regiones, e intermedia en la mayoría de los Servicios de Salud del país. Sin embargo, lo más relevante son las variaciones anuales, con frecuencia importantes, en casi todos ellos, explicables por una escasa población relativa ${ }^{19}$.

Chile presenta envejecimiento poblacional, lo cual influye junto con exceso de estadíos avanzados en la mortalidad creciente por este cáncer ${ }^{11}$, la cual se ha mantenido estable en los grupos bajo 50 años e incluso ha disminuido algo. Su aumento se ha producido en edades mayores de 50 años y especialmente sobre 60 , poblaciones que han aumentado en forma absoluta y proporcional ${ }^{10,19}$. Esto se observa en muchísimos países, atribuible a la transición demográfica ${ }^{20-24}$. La razón de este estudio es investigar su creciente mortalidad en poblaciones determinadas y su dependencia (hipótesis) de mayor edad y alta frecuencia mantenida de estadíos avanzados. 


\section{Material y Método}

Se revisa la literatura en relación a diferencias geográficas de incidencia y mortalidad por cáncer de mama en Chile y el mundo.

Este estudio se concentra en mortalidad por falta de un registro nacional, analizando el período 19902011 (22 años) en Chile, en el SSMSO (incluyendo incidencia) y en dos comunas de este Servicio de Salud, las de mayor población. Se desarrolla una comparación entre estos tres niveles: nacional, regional y local, utilizando cifras absolutas, tasas crudas, ajustadas según edad y por grupos quinquenales desde los 25 años, en forma anualizada. Se ha dispuesto de información para las siete comunas del SSMSO, pero se han analizado sólo las dos más pobladas: La Florida y Puente Alto.

Las fuentes de datos han sido el Departamento de Estadísticas e Información en Salud (DEIS), Ministerio de Salud y el Instituto Nacional de Estadísticas (INE), Ministerio de Economía. Esto a pesar de que desde los 70, la población chilena es sobreestimada por esta institución. Esto significa en Chile, especialmente en censo de 2002, un exceso poblacional importante, verificado al comparar los últimos censos decenales con las estimaciones INE, haciendo un ajuste entre el día censal de abril y su estimación al 30 de junio: nacidos vivos y fallecidos en el período de desfase correspondiente. Al parecer, el INE no ha valorizado la disminución creciente de natalidad, favorecida por el programa de planificación familiar iniciado en los 60 y por mayor educación femenina, entre otras razones. Sin embargo, se utilizan las estimaciones INE de población por ser oficiales. En realidad, las tasas de mortalidad verdaderas serían más altas.

\section{Resultados}

La evolución de la mortalidad por cáncer de mama a nivel nacional muestra un aumento constante de números absolutos y tasas desde comienzos del siglo XX hasta la década del 70. En la siguiente década hay un aumento brusco y extraordinario, de unas 8 veces según datos publicados ${ }^{13}$, debido a un probable error de registro o clasificación. En las dos últimas décadas se ha seguido produciendo en Chile un incremento importante de su mortalidad, del orden de $20 \%$ en sus tasas crudas o reales entre 1990 y 2011, subiendo desde 12-13 por $100.000 \mathrm{mu}-$ jeres hasta 15,7 el 2009 y 15,0 el 2011. El número absoluto de muertes se ha incrementado en forma constante, superando las 1.300 anuales (Figura 1).

La mortalidad por grupos quinquenales de edad muestra para el país, tasas muy bajas (entre 4 y 8 por 100.000) en las mujeres muy jóvenes, de 25-29 años, tasas que -con grandes oscilaciones anualestienden a aumentar en forma moderada. En cambio, las mujeres jóvenes entre 30 y 49 años presentan tasas mayores, crecientes según edad y que evolucionan a una disminución constante. Las mujeres de edad media entre 50 y 64 años tienen tasas bastante mayores (20-30 por 100.000) y también descienden, aunque menos marcadamente. Evolución estable en las mujeres entre 55 y 69 años (pero con tasas mayores de 30 a 50). Ocurre igual entre 69 y 79 años pero con tasas aún más elevadas (60-80 por 100.000). Sobre 80 años se aprecia también cierta estabilidad pero sus tasas son mucho más altas (100-140 por 100.000), lo cual dado el importante envejecimiento poblacional, explica el incremento creciente de la mortalidad por cáncer de mama en Chile (Figura 2).

En el SSMSO la mortalidad observada o real por

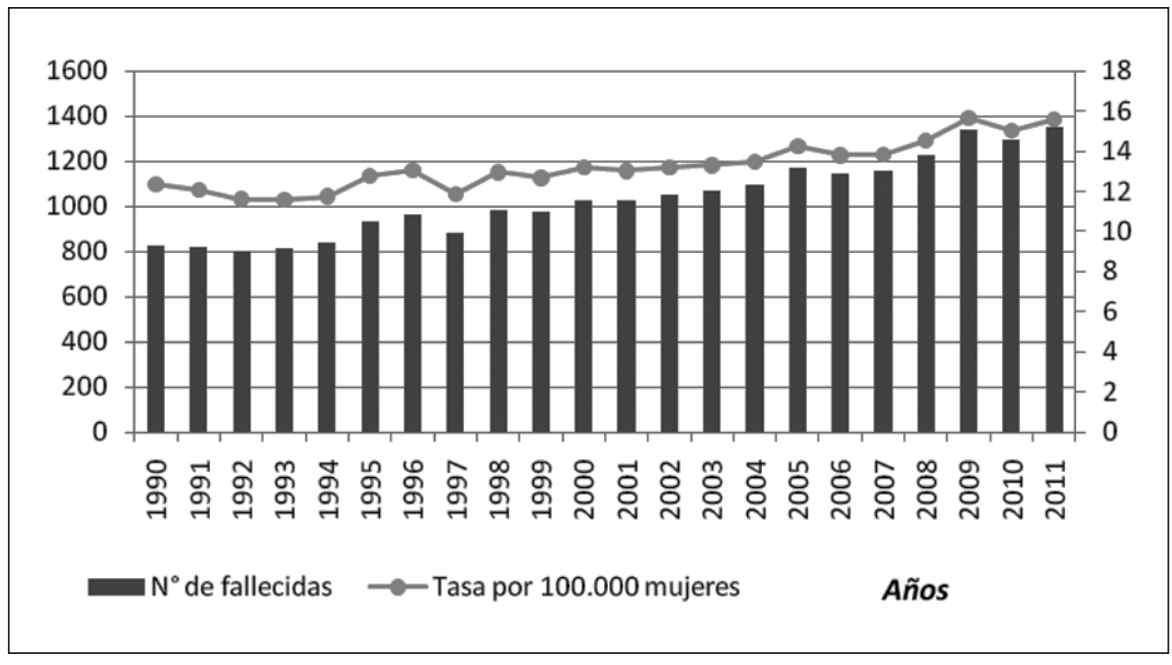

Figura 1. Número de fallecidas y tasas observadas de mortalidad por cáncer de mama en Chile, 1990-2011. 
CÁNCER DE MAMA EN CHILE. MORTALIDADES COMPARADAS: NIVELES NACIONAL, REGIONAL Y COMUNAL...
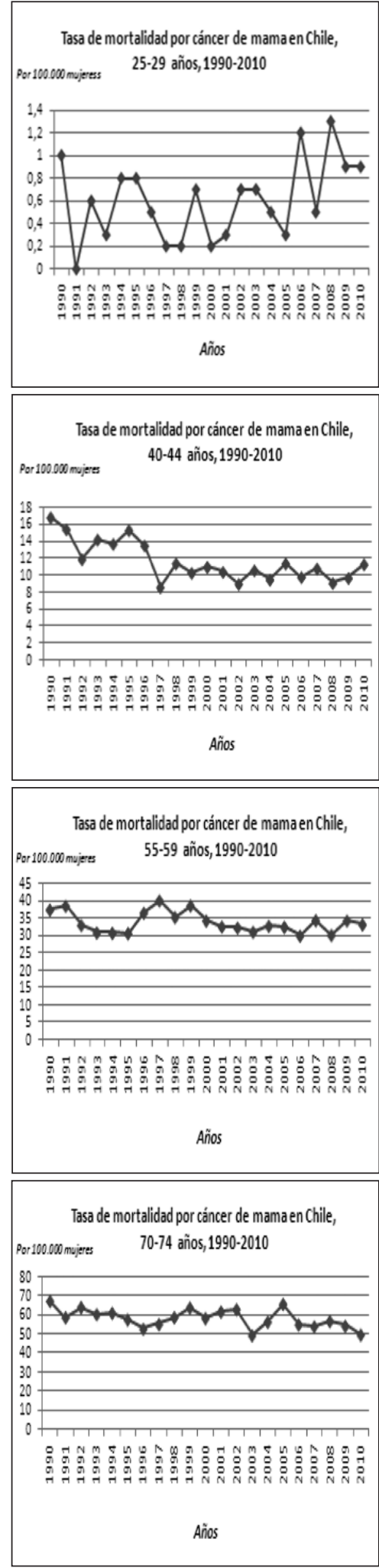
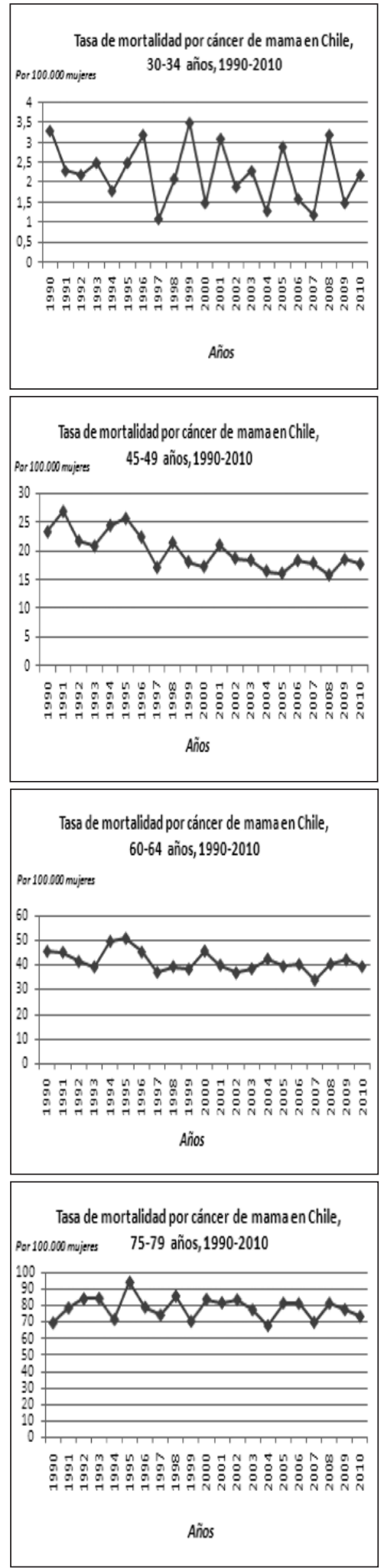
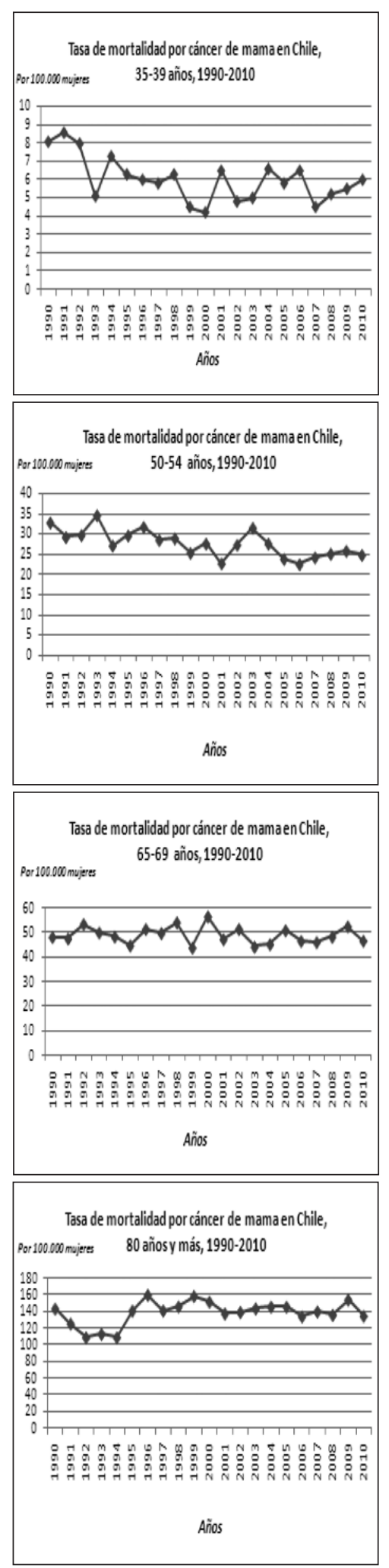

Figura 2. Tasas de mortalidad específicas por cáncer de mama según grupos quinquenales de edad Chile, 1990-2010. 

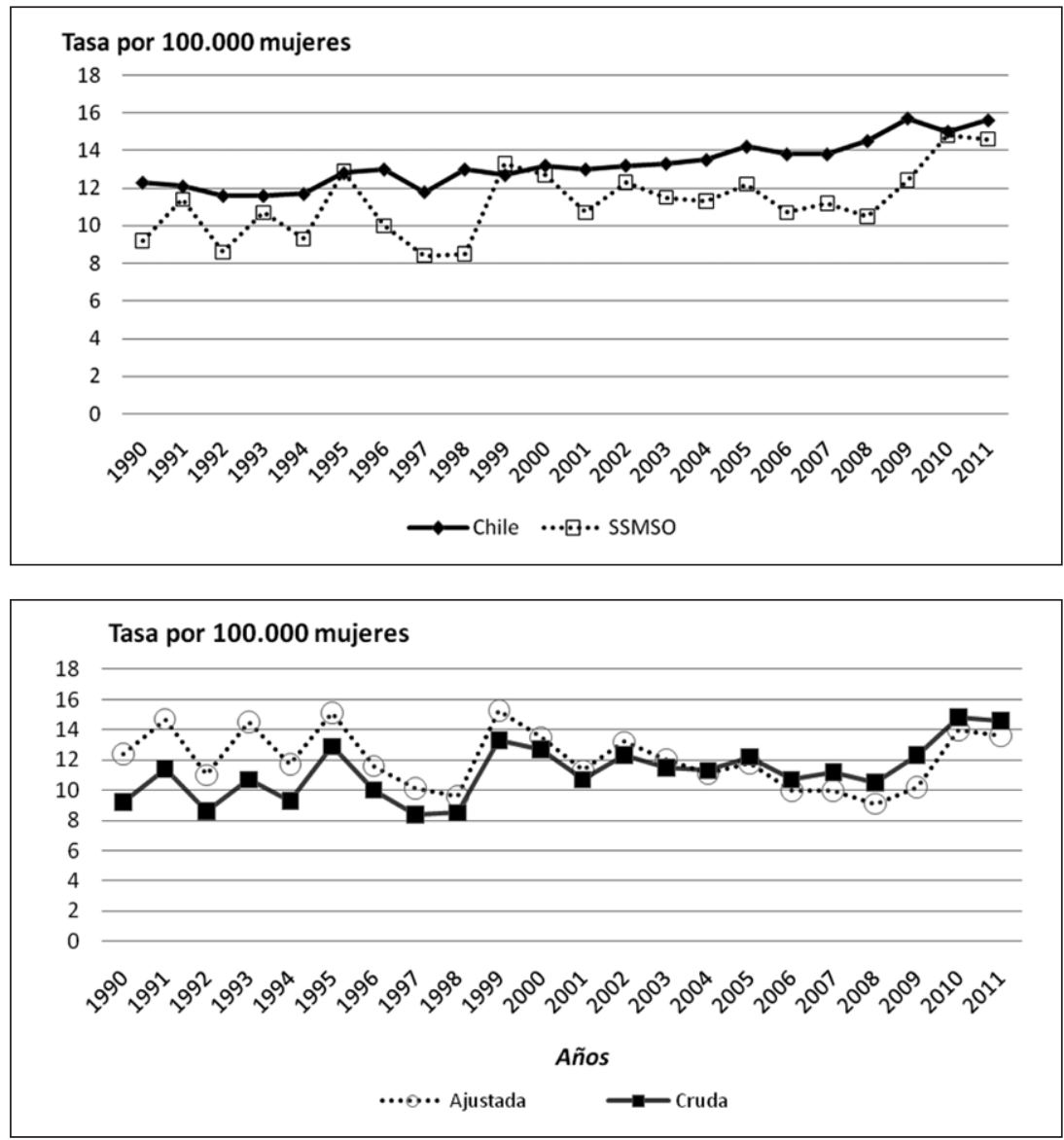

Figura 3a. Tasas crudas de mortalidad por cáncer de mama en Chile y SSMSO, 1990-2011.
Figura 3b. Tasas de mortalidad crudas y ajustadas por edad por cáncer de mama SSMSO, 1990-2011. cáncer de mama muestra en la década del 90 una tendencia al aumento, biológicamente significativa por ser el total de la población, con tasas entre 8 y 12 por 100.000 mujeres, aunque hubo alzas aisladas hasta de 13 por 100.000 . Su comparación con las tasas nacionales en el mismo período 1990-2011 muestra una situación favorable para el SSMSO, excepto los dos últimos años. En general aparece uno o dos puntos bajo la situación chilena (Figura 3a). El ajuste por edad en el SSMSO muestra una creciente similitud con las tasas crudas (Figura 3b).

En este Servicio de Salud, las tasas específicas por grupos quinquenales de edad presentan variaciones anuales, más importantes que lo observado a nivel nacional. El descenso de mortalidad tanto en las mujeres jóvenes como las de edad media es más marcado que en el país. Sin embargo, lo más relevante es que la base poblacional del SSMSO ha experimentado un incremento importante, especialmente en la última década. Sobre los dos fenómenos señalados: variaciones anuales y población muy aumentada se observa un descenso en la evolución temporal de las tasas en todos los grupos etarios, hasta los 69 años. En cambio, en las edades mayores su evolución en el tiempo muestra estabilidad. Las tasas anuales del SSMSO comparadas con Chile son inferiores en todos los grupos quinquenales de edad, salvo en las mujeres muy jóvenes en que son similares (Figura 4).

En el nivel comunal del SSMSO, Puente Alto y La Florida han tenido un crecimiento poblacional muy desigual: enorme en la primera y estancamiento en la segunda. En 1990 eran 128.525 y 168.245 mujeres respectivamente mientras el 2011 son 374.886 (más del doble) y 199.243 mujeres (sólo un 20\% de aumento). Las tasas observadas de mortalidad por cáncer de mama en estas dos comunas muestran una significativa diferencia en contra de La Florida, constante en todo el período, salvo una excepción en 1992 (Figura 5a). El ajuste por edad muestra en ambas comunas bastante similitud con las tasas crudas u observadas (Figura 5b). 
CÁNCER DE MAMA EN CHILE. MORTALIDADES COMPARADAS: NIVELES NACIONAL, REGIONAL Y COMUNAL...

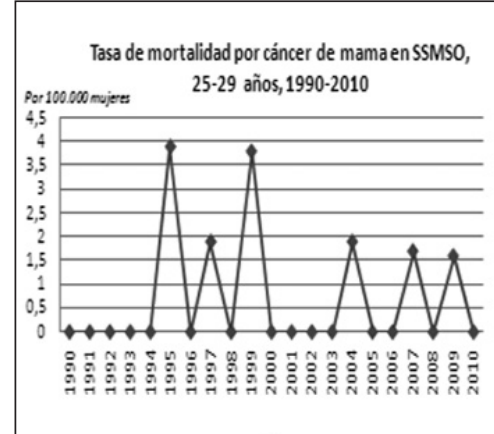

Años
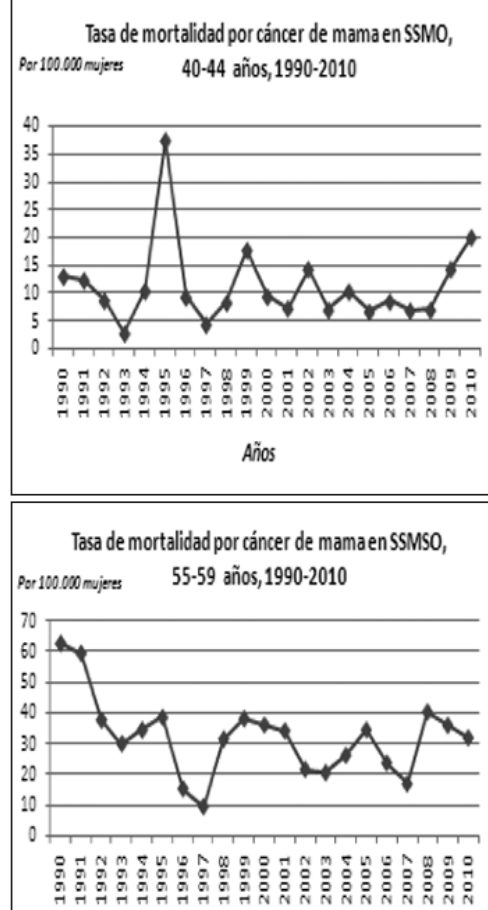

Años

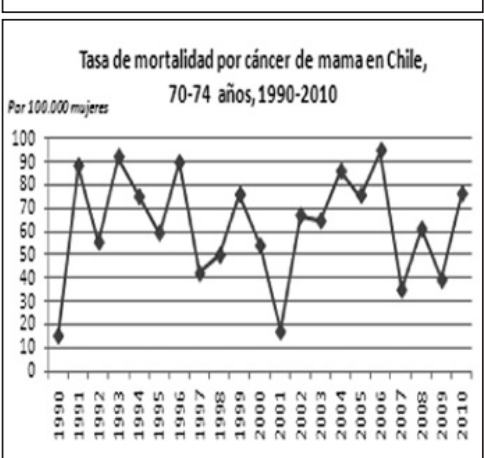

Años
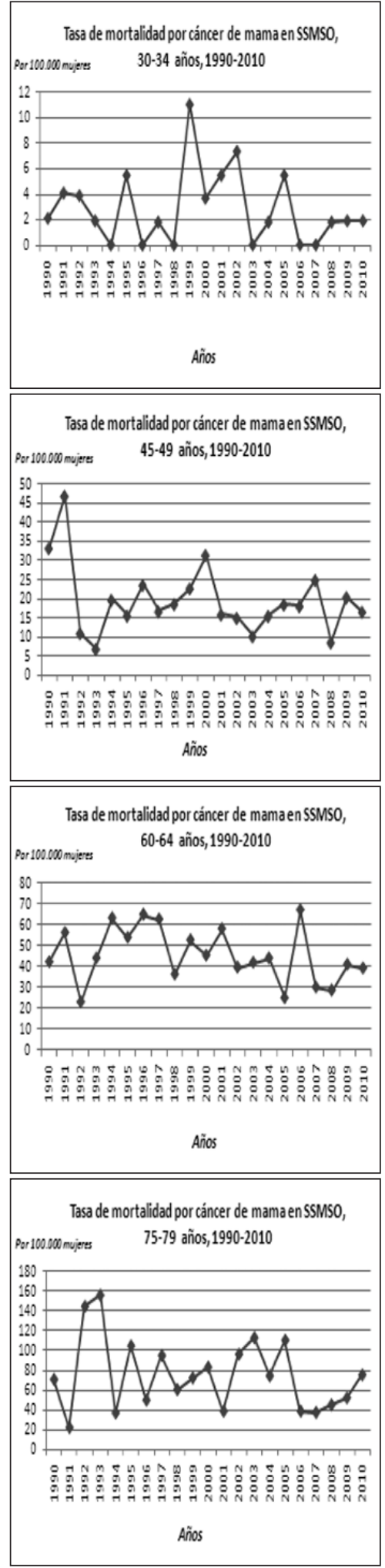

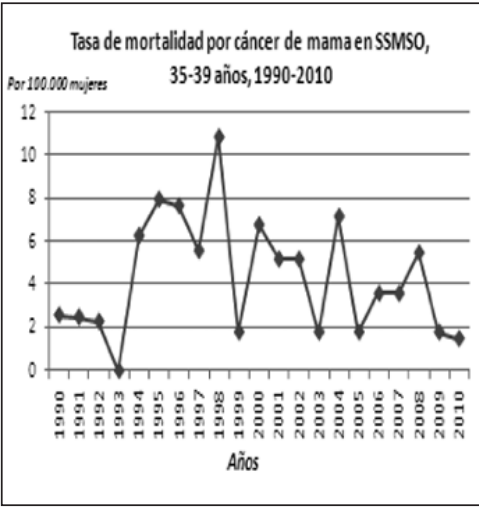

Tasa de mortalidad por cáncer de mama en SSMSO, 50-54 años, $1990-2010$
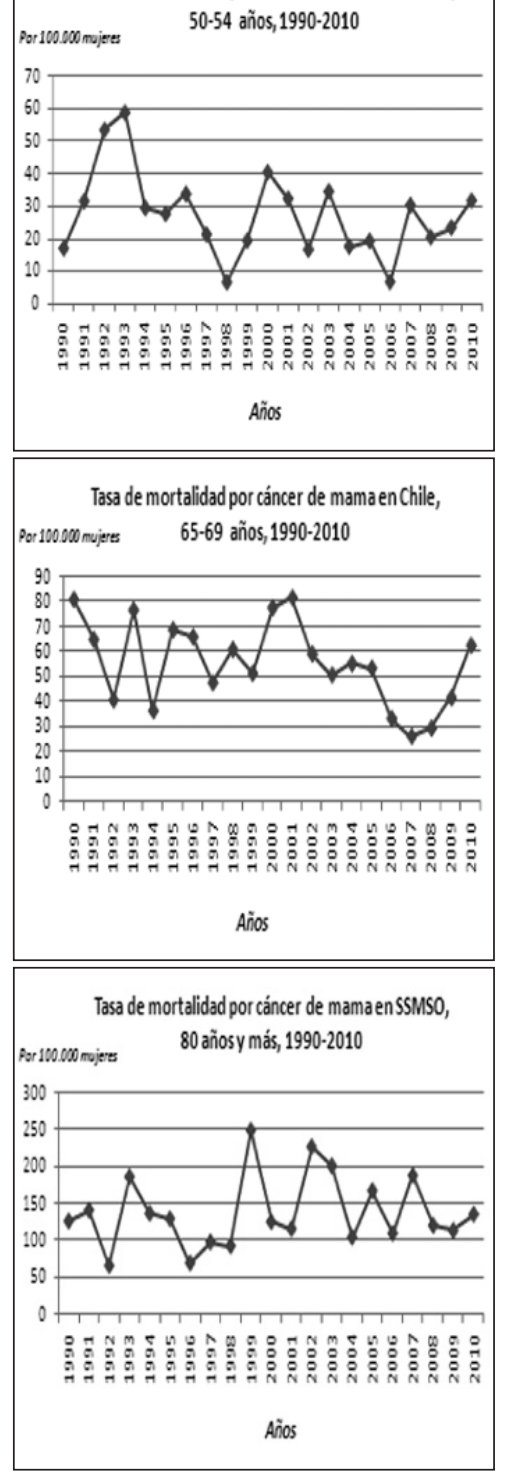

Figura 4. Tasas de mortalidad específicas por cáncer de mama según grupos quinquenales de edad en SSMSO, 19902010 . 


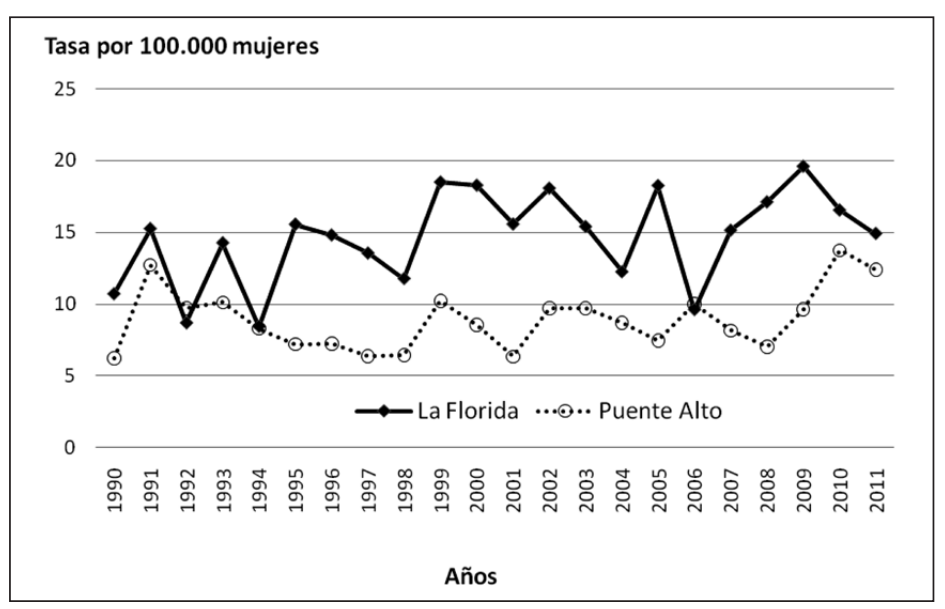

Figura 5a. Tasas crudas de mortalidad por cáncer de mama comunas de La Florida y Puente Alto, 1990-2011.
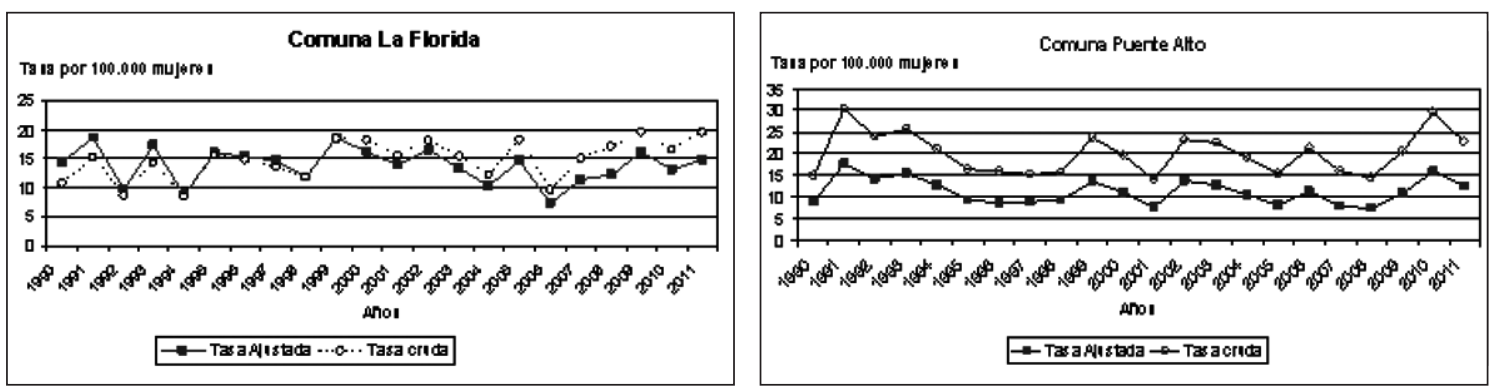

Figura 5b. Tasas crudas y ajustadas de mortalidad por cáncer de mama, comunas La Florida y Puente Alto, 1990-2011.

\section{Discusión}

Dada la confiabilidad de los datos de mortalidad en Chile, nuestro estudio se ha centrado en este indicador, con la fortaleza de aportar una comparación detallada entre el nivel nacional y el Servicio de Salud más poblado del país, además de dos de sus comunas. Nuestro registro del SSMSO tiene casi 20 años de antigüedad, 1994-2012 25 , respaldando una línea de investigación con varias publicaciones basadas en este registro ${ }^{10,11,26-29}$. Es poblacional por presentar tasas de incidencia altas, cercanas a 40 por 100.000 mujeres y superiores al nivel nacional. También, nuestro registro presenta, período 2006-2011, un $17 \%$ más de pacientes que los consignados en el nivel central ${ }^{26}$ aunque existe la salvedad de un $5 \%$ de pacientes atendidas pero residentes en comunas de otros Servicios de Salud ${ }^{26}$. Algunas pacientes nuestras son inicialmente atendidas en Servicios de Salud vecinos o instituciones privadas pero completan su atención en nuestro hospital o en el Padre Hurtado. Por lo señalado, es posible sostener que nuestro registro SSMSO es poblacional y no meramente hospitalario, además de confiable.
Una debilidad de esta investigación es la falta de correlación entre incidencia y mortalidad en el nivel nacional, la cual pudo hacerse sólo para el SSMSO y las dos comunas señaladas. Otra limitación es que nuestro registro de incidencia de este cáncer sería completo desde el 2006, derivado de la Reforma de Salud del 2005, AUGE-GES, que promovería por razones económicas la atención integral de acuerdo a la residencia real. De hecho, existe un claro aumento de incidencia a partir de ese año con estabilidad posterior (300 pacientes anuales) incluyendo hospitales Sótero del Río y Padre Hurtado, además del Centro de Referencia en Salud San Rafael, La Florida, con hospital Metropolitano.

Las diferencias observadas de mortalidad entre el nivel nacional y regional dependerían fundamentalmente de la diferente proporción de estadíos avanzados, III y IV junto con envejecimiento poblacional, confirmando la hipótesis ${ }^{10,26-29}$. El aumento de los números absolutos y de las tasas observadas o reales significa un problema de atención médica con tratamientos agresivos complejos, largos y costosos, tanto institucionales como para las pacientes, especialmente en senescentes y en estadíos avanza- 
$\operatorname{dos}^{30,31}$. Por eso es tan grave la alta proporción de estadíos avanzados en este cáncer en Chile, magnitud mantenida a pesar de la creencia contraria de algunos especialistas, influidos quizás por publicaciones que presentan tasas de mortalidad ajustadas por edad en vez de reales u observadas o que validan cambios bruscos de frecuencia sin fundamento epidemiológi$\mathrm{co}^{27,29}$. Por otra parte, podría influir el hecho de que el programa de prevención en este cáncer aparece como integral al rotularlo dirigido a las mujeres beneficiarias del sistema público desde los 15 años (edad en que no hay cáncer de mama) sin precisar lo más importante, su límite superior.

En realidad, el programa sugiere en alguna medida un límite superior (muy bajo) al establecer desde el 2011 una mamografía gratuita a los 50-54 años, con condiciones ${ }^{32}$. Este examen es oportunista y no de screening, palabra utilizada erróneamente en nuestro medio ya que no hay ni ha habido ningún programa de tamizaje en Chile sino sólo búsqueda de casos en poblaciones seleccionadas. Sin embargo, al ser esta edad tan baja se hace menos probable un diagnóstico oportuno de cáncer mamario en mujeres post-menopáusicas, las cuales tienen un riesgo mucho mayor. Ellas acuden regularmente a sus controles en la atención primaria por hipertensión arterial y diabetes mellitus y también por exámenes preventivos a cargo de enfermeras, significando una atención paralela a las matronas, responsables exclusivas hasta ahora de la pesquisa de cáncer de mama. Esto ocurre a pesar de que la expectativa de vida de la mujer chilena ya ha sobrepasado los 80 años $^{33,34}$. Por esta razón hemos insistido que el diagnóstico precoz de este cáncer debería incluir a las enfermeras, responsables habitualmente de los programas señalados en la atención primaria. Por lo demás, este nivel debería estar integrado con el hospitalario, una condición indispensable pero perdida desde hace tres décadas.

La situación epidemiológica del cáncer de mama en Chile comparándola con el SSMSO ofrece varios aspectos interesantes. En primer lugar, la menor mortalidad por este cáncer en este Servicio de Salud cuyas tasas son generalmente más bajas. También el ajuste por edad de sus tasas de mortalidad muestra gran similitud a las tasas observadas o crudas, significando que su población que siempre se ha caracterizado como muy joven estaría perdiendo esa condición, sea por el paso del tiempo o por inmigración ${ }^{34}$.

La mortalidad más alta en La Florida puede responder a su mayor incidencia $(38,7$ por 100.000 mujeres, período 2006-2011, versus 31,6 en Puente Alto), derivado quizás de un relativo mejor nivel socio-económico. La similitud creciente de tasas crudas y ajustadas en ambas comunas sugiere escasa influencia actual del envejecimiento, al igual que en el SSMSO, a diferencia del nivel nacional donde las tasas ajustadas por edad se han ido separando progresivamente de las crudas o reales ${ }^{29}$. Sin duda, el diferencial en mortalidad comparando estas dos comunas, amerita un estudio detallado dirigido a explorar variables como edad, grado de avance y tratamiento.

Quizás lo más relevante de este estudio es ratificar la influencia de una mayor edad y una alta frecuencia mantenida de estadíos avanzados en la mortalidad por este cáncer ${ }^{26-29}$. Son las mujeres senescentes, sobre 60 años y la alta proporción mantenida de estadíos III y IV, lo que marca la creciente mortalidad por cáncer de mama en Chile y ahora también en el SSMSO. La consecuencia es incumplimiento del gran objetivo declarado del programa nacional. Estas mujeres son quienes tienen las tasas más altas de incidencia y especialmente de mortalidad ya que con frecuencia son diagnosticadas en etapas avanzadas ${ }^{21,23,24}$. El mundo entero está destacando el relativo descuido del cáncer de mama en mujeres senescentes, situación que aparece cada vez con mayor énfasis en la literatura internacional ${ }^{35-38}$. Esta estrategia junto con otras acciones ha reducido significativamente la mortalidad por este cáncer en países desarrollados ${ }^{39}$. Su aplicación en Chie permitiría disminuir nuestra creciente mortalidad, a diferencia de lo que ocurre actualmente.

\section{Referencias}

1. Ferlay J, Parkin DM, Steliarova-Foucher E. Cancer incidence and mortality worldwide. Globocan 2008 IARC Cancer Base $\mathrm{N}^{\circ} 10$, http://globocan.iarc.fr

2. Ministerio de Salud, Nueva Zelanda. Estadísticas 19982006. En htpp.//www.moh.govt.nz/moh.msf/indexmh/ cancer-reg-cleaths-2006.

3. Ferlay J, Shin HR, Bray F, Forman D, Mathers C, Parkin DM. Estimates of cancer incidence and mortality in Europe in 2008. Eur J Cancer 2010;46:765-81.

4. Smigal C, Jemal A, Ward E, Cokkinides V, Smith R, Howe $\mathrm{Hl}$, et al. Trends in breast cancer by race and ethnicity: update 2006. CA Cancer J Clin. 2006;56:168-83.

5. De Angelis R, Tavilla A, Verdecchia A, Scoppa S, Hachey M, Feuer EJ, et al. Breast cancer survivors in the United States. Geographic variability and time trends 2005-2015. Cancer 2009;115:1954-66. On line: doi:10.1002/cner 24217.

6. Colditz GA. Epidemiology and prevention of breast cancer. Cancer Epidem Biomar. 2005;14:768-72.

7. Hankinson SE, Colditz GA, Willet WC. Towards an integrated model for breast cancer etiology: The lifelong interplay of genes, lifestyle, and hormones. Breast Cancer Res. 2004;6:213-8.

8. Deapen D, Liu L, Perkins C, Bernstein L, Ross RK. 
Rapidly rising breast cancer incidence rates among Asian-American women. Int J Cancer 2002;99:747-50.

9. Roa I. La necesidad de crear registros de cáncer. Rev Chil Cir. 2002;54:206-11.

10. Serra I, Martínez R, Mimica X, Aguayo C. ¿Es posible disminuir la mortalidad por cáncer de mama en Chile? Análisis epidemiológico en la Región Metropolitana, 2000-2007. Rev Chil Cir. 2010;62:339-47.

11. Serra I, Martínez R, Mimica X, Cavada G, Aguayo C. Cáncer de mama en Chile. Un aporte clínico y epidemiológico según un registro poblacional metropolitano: 1.485 pacientes. Rev Chil Cir. 2009;61:507-14.

12. Globocan 2008 IARC. Cancer Base $N^{\circ} 10$. En htpp:// www.minsal.cl.

13. Medina E, Marré E, Irarrázaval M, Kaempfer AM. Cáncer de mama en Chile. Rev Med Chile 1981;109:88796.

14. Castillo B, Solís F, Kaempffer AM. Cáncer de mama: atención de salud y nivel de vida en la mortalidad prematura. Rev Chil Obstet Ginecol. 1994;59:108-15.

15. Pierart J, Lagos O, Cid L, Guijarro P, Martínez A, Santander P. Mortalidad por cáncer de mama en la VIII Región de Chile. Rev Chil Cir. 1996;48:608-18.

16. Armijo R. The epidemiology of cancer in Chile. Natl Cancer Inst Monogr. 1979;53:115-8.

17. Castillo B, Mardones G. Certificación médica de las defunciones en los Servicios de Salud de Chile. Rev Med Chile 1986;114:693-700.

18. Núñez ML, Icaza MG. Calidad de las estadísticas de mortalidad en Chile, 1997-2003. Rev Med Chile 2006;134:1191-6.

19. Ministerio de Salud, Chile. Departamento de Estadísticas e Información de Salud. Defunciones por tumor maligno de la mama femenina por servicios de salud y grupos de edad 1990-2008.

20. Szot J. La transición demográfico-epidemiológica en Chile, 1960-2001. Rev Esp Salud Pública 2003;77:60513. En internet: Scielo, ISSN 1135-5727.

21. Mazzafero V, Masse G. La transición demoepidemiológica en Europa y América Latina. Rev Inst Hig Med Soc. 1999;3:9-20.

22. López-Ríos O, Lazcano-Ponce EC, Tovar-Guzmán V, Hernández-Avila M. La epidemia de cáncer de mama en México: ¿consecuencia de la transición demográfica? Salud Publ Mex. 1997;39:259-65.

23. Lozano-Ascencio R, Gómez-Dantés H, Lewis S, TorresSánchez L, López-Carrillo L. Tendencias del cáncer de mama en América Latina y El Caribe. Salud Publ Mex. 2009;51(suppl 2):S147-S156.

24. Salas C, Grisales H, Calidad de vida y factores asociados en mujeres con cáncer de mama en Antioquia,
Colombia. Rev Panam Salud Publ. 2010;28:9-18.

25. Aguayo C. Creación y explotación de un registro poblacional y hospitalario de cánceres del adulto en el Servicio de Salud Metropolitano Sur Oriente, 20012005. Tesis Magíster en Salud Pública, Escuela de Salud Pública, Universidad de Chile, 2007, 102 pág.

26. Martínez R, Serra I, Aguayo C, Maya D, Vásquez A. Cáncer de mama: Incidencia y características en 3.207 pacientes según registro poblacional, Servicio de Salud Sur Oriente, 1994-2011. 85 Congreso Chileno de Cirugía. Libro de Resúmenes, pág. 108.

27. Serra I. Cáncer de mama en Chile: pasado, presente y futuro. Artículo editorial. Rev Chil Cir. 2011;63:5536.

28. Serra I, Maya D, Martínez R, Decinti E. Cáncer de mama en Chile. Tardanzas en su atención médica. Cuad Med Soc. 2012;51:54-65.

29. Serra I, Martínez R, Reyes G, Sierra P, Aguayo C. Envejecimiento y alta prevalencia de estadíos avanzados determinan la creciente mortalidad por cáncer mamario en Chile. Rev Chil Cir. 2012;64:137-46.

30. Orellana C, Torres S, Derio L, Prieto M. Cancer care in Chile. Lancet Oncol. 2003;4:653-6.

31. Hacket TP, Cassem NH, Raker JW. Patient delay in cancer. New Eng J Med. 1973;289:14-20.

32. Castillo L. Aprueba programa de imágenes diagnósticas en atención primaria. Resolución Exenta No 52, Subsecretaría de Salud, 11 Febrero 2011.

33. Arteaga O, Thollaug S, Nogueira AC, Darras C. Información para la equidad en salud en Chile. Rev Panam Salud Publica 2002;11:374-385.

34. Albala C, Sánchez H, Lera L, Angel B, Cea X. Efectos sobre la salud de las desigualdades socioeconómicas en el adulto mayor. Rev Med Chile 2011;139:1276-85.

35. Crivellari D, Aapro M, Leonard L, von Minckwitz G, Brain E, Goldhirsch A, et al. Breast cancer in the elderly. J Clin Oncol. 2007;25:1882-90.

36. Paesmans M, Ameye L, Moreau M, Rozenberg S. Breast cancer screening in the older woman: An effective way to reduce mortality? Maturitas 2010;66:263-7.

37. Chu KC, Smart CR, Tarone RE. Analysis of breast cancer mortality and stage distribution by age for the Health Insurance Plan clinical trial. J Natl Cancer Inst. 1988;80:1125-32.

38. Altekruse SF, Kosary CL, Krapcho M, Neyman N, Aminou R, Waldron W, et al. Breast cancer risk by age. SEER Cancer Statistics Review, 1975-2007. En htpp:// seer.cancer.gov/csr/1975 2007/.

39. Peto R, Boreham J, Clarke M, Davis C, Beral V. UK and USA breast cancer deaths down $25 \%$ in year 2000 at ages 20-69 years. Lancet 2000;355 (9217):1522. 